\title{
Obstetric Anaesthesia and Analgesia Related Complications in Czech and Slovak Republics: National Observational Survey in 2015
}

\author{
Štourač P. ${ }^{1}$, Bláha J. ${ }^{2}$, Nosková P. ${ }^{2}$, Grochová M. ${ }^{3}$, Firment J. ${ }^{3}$, Schwarz D. ${ }^{4}$, \\ Richterová S. ${ }^{5}$, Seidlová D. ${ }^{6}$, Klozová R. ${ }^{7}$ and OBAAMA-INT Study Group
}

官OBAAMA-INT

\begin{abstract}
${ }_{1}^{1}$ Department of Paediatric Anaesthesiology and Intensive Care Medicine, Medical Faculty of Masaryk University, Faculty Hospital Brno
${ }^{2}$ Department of Anaesthesiology and Intensive Care Medicine, 1st Medical Faculty of Charles University in Prague, General University Hospital in Prague

3l. Department of Anaesthesiology and Intensive medicine, University Teaching Hospital of Louis Pasteur and University of Pavol Joseph Safarik, Medical Faculty

${ }^{4}$ Institute of Biostatistics and Analyses, Medical Faculty of Masaryk University

${ }^{5}$ Department of Anaesthesiology and Intensive Care Medicine, University Hospital Martin, Jessenius Faculty of Medicine, Comenius University

${ }^{6}$ Department of Anaesthesiology and Intensive Care Medicine, $2^{\text {nd }}$ Medical Faculty of Charles University in Prague, Teaching Hospital in Motol

${ }^{7} 2^{\text {nd }}$ Anaesthesiological Department, University Hospital Brno
\end{abstract}

\section{Background and Goal of Study}

In the 2011 year OBAAMA-CZ study described anaesthesiological practice for obstetric anaesthesia and analgesia in the Czech Republic. One of the objectives of the OBAAMA-INT study performed in the year 2015 was to describe obstetric anaesthesia and analgesia related complications in the Czech (CZ) and Slovak Republics (SR).

\section{Materials and Methods}

OBstetric Anaesthesia and Analgesia Month Attributes International (OBAAMA-INT) was held on anaesthetic departments throughout the CZ and SR. With Ethical Committees Approval we aimed to enroll all 149 obstetric departments in CZ and SR and to monitor every case and analgesia related complications during November 2015. Data were recorded to Case Report Form with two parts (Demography 2014 and Case Report). Data were registered with the use of CLADE-IS Electronic Data Capture system (Institute of Biostatistics and Analyses, Ltd.) and described descriptively (incidence, mean, median, SD).

\section{Results and Discussion}

During the study period, we enrolled 105 participating centers (70 in CZ; 35 in SR) and 3590 valid cases. Caesarean Section (CS) was recorded in 2548 cases (71.0\%), 1158 cases (31.4\%) were obstetric analgesia and 181 cases $(5.0 \%)$ were early postpartum procedures. The most preferred type of anaesthesia for CS was neuraxial anaesthesia (62.5\%); spinal in $87.5 \%$. Most frequent complications related to general anaesthesia were difficult airways $(1.4 \%)$ at the induction, hypertension $(7.9 \%)$ and tachycardia (5.2\%) during the anaesthesia. Complications related to spinal or epidural anaesthesia were more than 1 attempt to achieve neuraxial space $(23.6 \% ; 16.1 \%)$, blood in the needle $(1.9 \% ; 1.0 \%)$, paresthesia $(1.3 \% ; 1.0 \%)$ and high blockade $(0.4 \% ; 0.5 \%)$. Epidural labor analgesia rate was $11.1 \%$. For epidural labor analgesia the most frequent complication was more than 1 attempt to achieve epidural space $(15.5 \%)$, blood in the needle or catheter (3.1\%) and unattended dural puncture $(0.7 \%)$. Postpuncture headache was recorded in $1.2 \%$ overall. In case of epidural labor analgesia it was more frequently associated with multiple attempts $(p=0.007)$ and unattended dural puncture $(p<0.001)$

\section{Conclusion}

There are no previously published Czech and Slovak national data available, hence presented data are very important starting point for future improvement.

\section{Acknowledgement}

Financial Support Grant Identifier: CSARIM201501.

ClinicalTrials.gov Identifier: NCT02380586

\section{Endorsement}

Czech Society of Anaesthesiology and Intensive Care Medicine Slovak Society of Anaesthesiology and Intensive Care Medicine of anaesthetic care in peripartum period and all anaesthesia

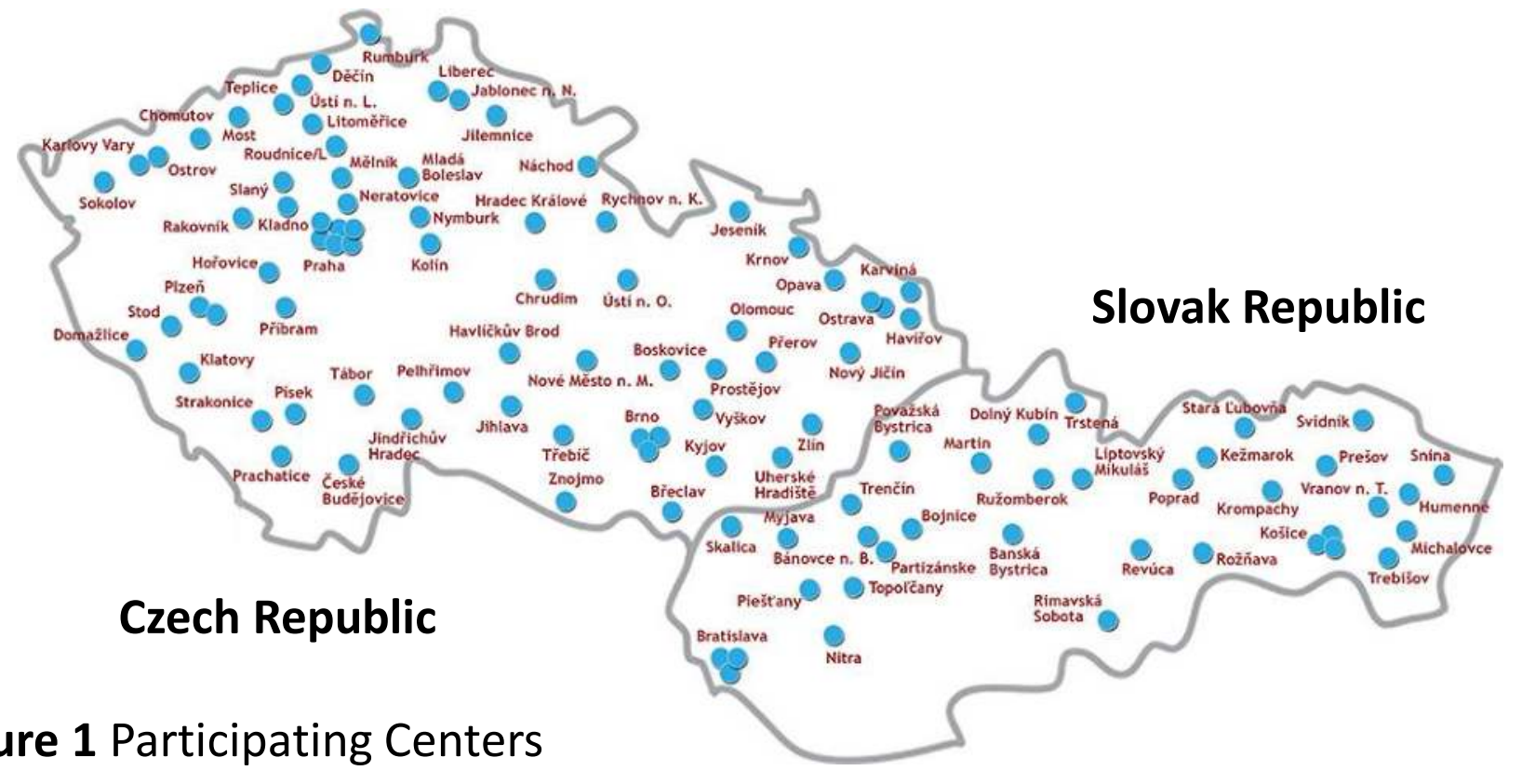

General Anaesthesia

\begin{tabular}{lccc}
\hline & Total & $\mathrm{CZ}$ & $\mathrm{SR}$ \\
\hline Induction (N) & 956 & 672 & 284 \\
Regurgitation & $0.1 \%$ & $0.1 \%$ & $0.0 \%$ \\
Hyposaturation & $0.3 \%$ & $0.1 \%$ & $0.7 \%$ \\
Difficult Airway & $1.4 \%$ & $1.5 \%$ & $1.1 \%$ \\
Other & $0.2 \%$ & $0.1 \%$ & $0.4 \%$ \\
Course (N) & 956 & 672 & 284 \\
Hypertension & $7.9 \%$ & $9.1 \%$ & $5.3 \%$ \\
Hypotension & $1.3 \%$ & $1.2 \%$ & $1.4 \%$ \\
Tachycardia & $5.2 \%$ & $4.8 \%$ & $6.3 \%$ \\
Bradycardia & $0.4 \%$ & $0.3 \%$ & $0.7 \%$ \\
Arrhytmia & $0.5 \%$ & $0.3 \%$ & $1.1 \%$ \\
Hyposaturation & $0.5 \%$ & $0.1 \%$ & $1.4 \%$ \\
Other & $0.8 \%$ & $0.7 \%$ & $1.1 \%$ \\
\hline
\end{tabular}

Figure 2 Obstetric Anaesthesia Related Complications

\section{OBAMA-INT Study Group}

Czech Republic
MUDr. Vladimír Zemánek (Boskovice); MUDr. Hana Harazim, MUDr. Martina Kosinová, MUDr. Kateřina Vajčnerová, MUDr. Dagmar Seidlová, Ph.D. (Brno); MUDr. Vladimír Hluchý (Břeclav); MUDr. Jan Cepák (České Budějovice); MUDr. Nikola Benešovã (Děč́n); MUDr. Vladislav Cerný (Domažlice); MUDr. Robert Bocek (Haviřov); MUDr. Jitka Mannová, Ph.D. (Havličǩiv Brod) MUDr. Jiř Steinbach (Hořovice); MUDr. Xénia Silová (Hradec Králové);MUDr. Jan Němec (Chomutov); MUDr. Martin Zemánek (Chrudim) MUDr. Petr Kudrna (Jesenice); MUDr. Petr Horáček, MUDr. Bohdan Trnka (Jihlava); MUDr. Gabriela Pohořalá (Jilemnice) MUDr. Ladislav Rychtárik (Jindrichüv Hradec); MUDr. Zdenêk Kos, DEAA (Karlovy Vary); MUDr. Radmila Vojkưvková (Karvina); MUDr. Kamil Kodras (Kladno); MUDr. Mirram Gredová (Klatovy); MUDr. Jirí Blazek (Kolin); MUDr. Slavka Stajančová (Krnov); MUDr.

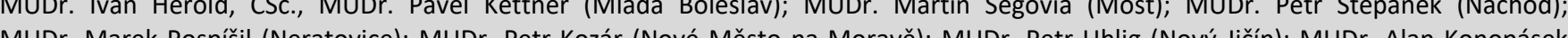
(Nymburk): MUDr. Zdeněk Mrozek, Ph.D. (Olomouc); MUDr. Božena Jě̌ová (Opava): MUDr. Hana Durdová (Vítkovice): MUDr. Christian Kufa MUDr. Blazena Zaoralová (Ostrava); MUDr. Ivana Jarośová (Ostrov); MUDr. Frantǐ̌ek Bárta (Pelhrimov); MUDr. Tomáš Piksa (Písek): MUDr. Roman Bosman, MUDr. Roman Sviták (Plzeň); MUDr. Jaroslava Ščamburová, MUDr. Jan Bláha, Ph.D., MUDr. Pavlina Nosková, MUDr. Valter Zenkner, DEAA, MUDr. Markéta Kopecká, MUDr. Zdeňka Krupková, MUDr. Jiř́ Roškot (Praha): MUDr. Petr Rǔžčcka (Prachatice); MUDr. Gréta Směšná (Prostějov); MUDr. Petr Dušek (Přerov); MUDr. Jana Popieluchová (Přibram); MUDr. Pavel Padrta (Rakovnik); MUDr. Pavel Fiala (Roudnice nad Labem); MUDr. Jan Bělič (Rumburk); MUDr. Aleš Vlček (Rychnov nad Kně̌̌nou); MUDr. Jirí Dvorăk (Slaný); MUDr. Alexander Aboši (Sokolov); MUDr. Vladislav Kř́ż (Stod); MUDr. Marek Zbořil (Strakonice); MUDr. Radovan Prchlik (Tabor); MUDr. Barbora Nêmcová (Teplice); MUDr. Simona Hnátová (Třebic); MUDr. Irena Šnajdrová (Uherské doc. MUDr. Tomáš Gabrhelik, Ph. D. MUDr. Lubomír Večěra (Zlin). MUDr. Iva Šprinclová (Znojmo) Slovak Republic

MUDr. Juraj Kusý (Bánovce nad Bedravou); MUDr. Diana Valčuhová (Banská Bystrica); MUDr. Mária Šramková, PhD. (Bojnice). MUDr. Renata Tobolaková MUDr. Viera Lesná, MUDr. Jaroslava Macková, MUDr. Lucia Várošová (Bratislava). MUDr. František Mičan (Dolný Kubín); MUDr. Sergej Suško (Humenné); MUDr. Serhiy Rak (Kežmarok); doc. MUDr. Jozef Firment, PhD., MUDr. Monika Grochová, PhD., MUDr. Jana Simonova, PhD., MUDr. Gabriela Mizlova, MUDr. Marcela Slukova,, MUDr. Ján Slávik, MBA, MUDr. Pavol Rusnák (Košice); MUDr. Zuzana Baluchová (Krompachy); MUDr. Dávid Druska, MUDr. Mária Buffová (Liptovský Mikuláš); MUDr. Stanislava Richterová, PhD. (Martin); MUDr. Lucia Holúková, MUDr. Marta Tkáčová (Michalovce); MUDr. Peter Mokoš (Myjava);

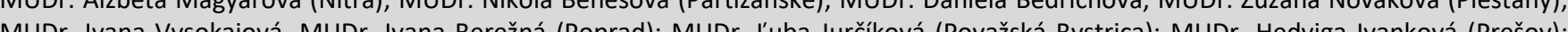

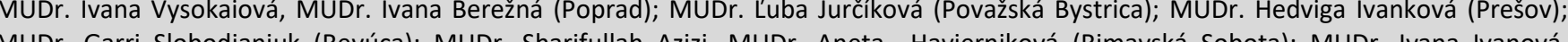
MUDr. Zsolt Varga (Rožňava); MUDr. Marián Paulík (Ružomberok): MUDr. Ivo Horský (Skalica); MUDr. Andrea Hennelová (Snina):

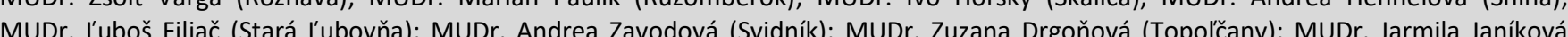
(Trebišov); MUDr. Lubica Mišáková (Trenčin); MUDr. Božena Horanová (Trstená); MUDr. Lucia Tesáková (Vranov nad Toplou) IBA LF MU

Doc. Ing. Daniel Schwarz, Ph.D., RNDr. Jiří Jarkovský, Ph.D., Mgr. Jan Švancara, Mgr. Hana Zelinková

Lucia Macková, Eliška Hamřiková, Ondřej Hrůza, Jiří Libra

\section{FAKULTNINICE BRNO}
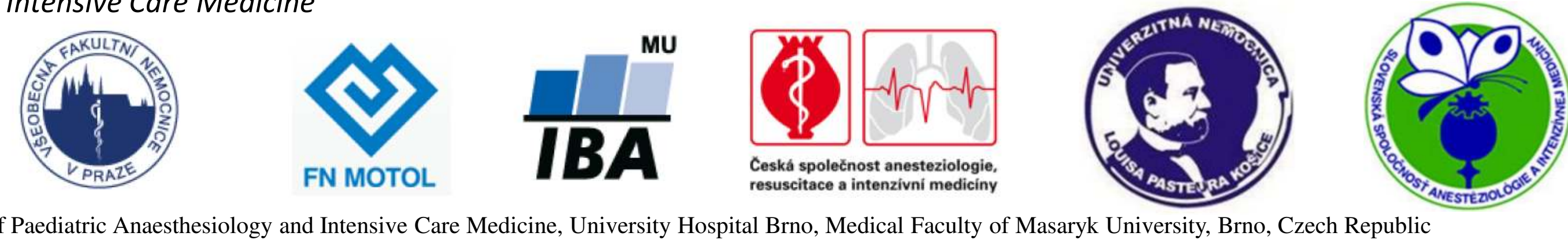